\title{
Cluster of Legionnaires' disease in a newly built block of flats, Denmark, December 2008 - January 2009
}

L H Krøjgaard (lkd@ssi.dk) ${ }^{1,2}$, K A Krogfelt ${ }^{1}$, H J Albrechtsen², S A Uldum

1. Department of Microbiological Surveillance and Research, Statens Serum Institut, Copenhagen, Denmark

2. Department of Environmental Engineering, Technical University of Denmark, Lyngby, Denmark

Krøjgaard LH, Krogfelt KA, Albrechtsen HJ, Uldum SA. Cluster of Legionnaires' disease in a newly built block of flats, Denmark, December 2008 - January 2009 . Euro Surveill. 2011;16(1):pii=19759. Available online: http://www.eurosurveillance.org/ViewArticle.aspx?Articleld=19759

During December 2008 to January 2009, two persons contracted Legionnaires' disease in a newly built block of flats in a suburb of Copenhagen in Denmark. Polymerase chain reaction and culture was used to diagnose Legionnaires' disease in this cluster. Isolates from both patients tested positive for Legionella pneumophila serogroup 1 subgroup Philadelphia sequence type 1 and the same strain was detected in hot water samples taken from the residential area indicating that the hot water supply system was the most likely source of infection. Legionella was not detected in the cold water. Two interventions were conducted to limit the Legionella colonisation of the piping and storage tanks and the effect was monitored by investigating water samples from various sites in the block of flats. Only the second intervention had a sufficient effect on the Legionella colonisation. The cluster described here points to several risk factors regarding growth of Legionella in hot water systems: (i) stagnancy of water from when the building is constructed and piping installed and until residents move in, (ii) stagnancy and low temperature (from room temperature to approximately $38{ }^{\circ} \mathrm{C}$ ) of water in shower hoses and (iii) failure in operation of and control measures for the hot water system.

\section{Introduction}

Legionnaires' disease (LD) is a severe pneumonia with high mortality caused by the inhalation of aerosolised Legionella bacteria. Legionella occurs naturally in water sources but the bacteria multiply to high numbers at temperatures between $20^{\circ} \mathrm{C}$ and $45^{\circ} \mathrm{C}$ [1]. The multiplication of Legionella is, associated with several other factors apart from water temperature such as water being stagnant, type of pipe material used, the presence of a biofilm (a micro-environment between surface and water) and amoebae $[1,2]$. These factors are the reasons why man-made water systems, often harbour Legionella in high numbers. Outbreaks of LD are often associated with aerosols from cooling towers $[3,4]$, spas $[1,5,6]$, and hot and cold water systems at hospitals [7,8] and hotels [9].Studies have shown that domestic hot water systems are often colonised with
Legionella $[10,11]$ but outbreaks are rarely associated with potable water distribution systems.

Each year 100-130 cases of LD are notified in Denmark (approximately 20 per million) which is a rather high incidence compared to other European countries [12]. Most of the cases are sporadic and only few outbreaks have been identified. This study was conducted to investigate factors associated with risk of Legionella colonisation in new buildings and to monitor and investigate the effect of control measures.

\section{Cluster description}

A cluster of two culture-confirmed LD cases was identified during December 2008 to January 2009 in a suburb of Copenhagen in Denmark. Neither case had any recent history of travel. On 11 November 2008, the first case (Case 1), a man in his early forties with an underlying condition, was hospitalised $250 \mathrm{~km}$ away from the building that was later found to be the source of infection. Case 1 was linked to the block of flats only after the second case (Case 2) was diagnosed, since he had only spent a few days in the newly built block of flats, in an apartment which had not been used before. On 30 December the second patient (Case 2), a man in his mid-sixties who had been treated for an underlying condition, was hospitalised and on 5 January 2009 he was diagnosed with LD. He died 20 days after admission. Case 2 lived in the building later found to be the source of infection. His family had earlier complained about the low temperature of the hot water in the apartment and the hot water of this apartment was therefore the first to be investigated.

Both cases were positive for L. pneumophila by polymerase chain reaction (PCR) on samples from tracheal secretions and L. pneumophila was subsequently isolated by culture by standard techniques. Isolates were identified by agglutination test (Legionella latex test DRo80oM, Oxoid); and sero- and subgrouping were performed with monoclonal antibodies [13]. Extracted DNA was analysed by sequence-based typing (SBT) according to the European Working Group for Legionella Infections (EWGLI) standard 
procedure [14]. Both cases were found to be infected with L. pneumophila serogroup 1 subgroup Philadelphia sequence type (ST) 1.

All residents of the block of flats, as well as visitors, were informed about the outbreak and advised to go and see their general practitioner and take blood samples if showing symptoms of LD and 16 of these chose to do so. These 16 samples were tested for Legionella antibodies. Three of them were also tested for Legionella urinary antigen but none of the samples were positive. None had pneumonia but some may have had Pontiac fever based on clinical symptoms (influenza-like illness caused by Legionella infection), although this was not confirmed by laboratory tests.

\section{Methods}

The water system

The building identified as the most probable source of contamination had 225 apartments distributed in six blocks. Of these 225 apartments, 210 were inhabited at the time the cluster was detected. The hot water system had two boilers in use and a circulation pump in place to circulate the hot water. In each stairway, proportional thermostatic adjusting valves set at $50{ }^{\circ} \mathrm{C}$ regulated the flow of hot water. The water supply in the area was based on ground water, which was not disinfected and distributed without disinfection residuals.

\section{FIGURE 1}

Concentration of Legionella spp. in the hot water system (B-samples ${ }^{\mathrm{a}}$ ), data collected from seven different apartments, Copenhagen, Denmark, January - September 2009

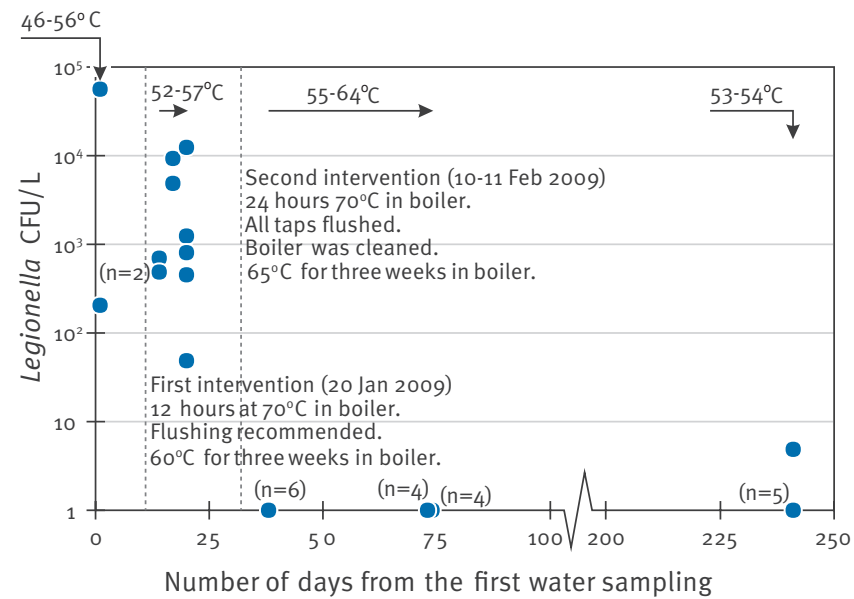

CFU: colony forming unit.

a One litre samples collected after flushing until constant water temperature (warm or cold) was reached.

Concentration of Legionella spp over time in the hot circulation water in the block of flats. The water samples were collected after flushing until constant temperature (B-samples), and the temperature interval in the samples in the different apartments are given at the top of the figure. Each dot represents one sample but there are two samples with 800 CFU/L on day 20 , six samples with no Legionella detected on day 38 , four samples with no Legionella detected on day 73 , four samples with no Legionella detected on day 74 and five samples with no Legionella detected on day 241. The dotted lines indicate the first and the second intervention, respectively. The first water samples were collected on 9 January 2009.
Sampling and analysis

Water was collected from the building on eight occasions from 9 January to 7 September 2009. Two types of water samples were collected each time from kitchen and bathroom taps as well as from shower hoses: A-samples - the first litre of water (first flush) - and B-samples - one litre collected after flushing until constant water temperature (warm or cold) was reached. Apartments located as distant from and as close to the boiler as possible and apartments with no, low or normal levels of water consumption, as well as apartments associated with the cases, were sampled. B-samples from shower hoses all had a temperature of $38{ }^{\circ} \mathrm{C}$ due to the thermostatic mixing valves installed on shower fixtures in all apartments.

The temperature ranges in the samples in the different apartments are given at the top of the figure. The first water samples were collected on 9 January 2009. Unless otherwise indicated, each dot represents one sample. The dotted lines indicate the two interventions.

The first water samples were collected on 9 January 2009. Unless otherwise indicated, each dot represents one sample. The dotted lines indicate the two interventions.

Testing of water samples for the detection of environmental Legionella by cultivation was done according

\section{FIGURE 2}

Concentration of Legionella spp. in the first litre of water sampled (A-samples $\left.{ }^{\mathrm{a}}\right)$, data collected from seven different apartments, Copenhagen, Denmark, January - September 2009

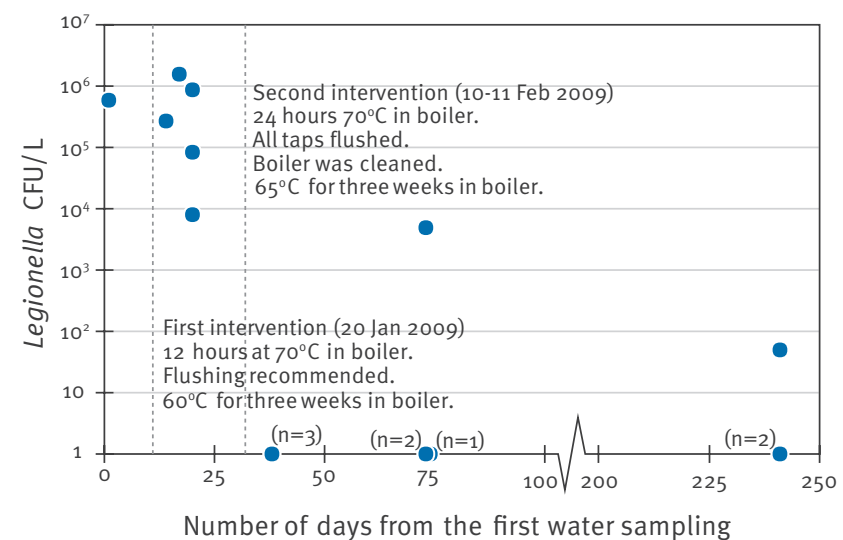

CFU: colony forming unit.

a A-samples are the first litre of water from the shower hose (first flush).

Concentration of Legionella spp over time in the first litre of water sampled (A-samples) from different shower hoses. There are 3 samples on day 38 , where no Legionella could be detected. Each dot represents one sample but there are two samples on day 73 and one sample on day 74 where no Legionella could be detected. At day 241 , there are two samples with no Legionella detected. The dotted lines indicate the first and the second intervention, respectively. The first water samples were collected 9 January 2009. 
to the ISO standard 11731:1998 [26]. One-litre water samples were concentrated 10 -fold and 100-fold respectively by $0.2 \mu \mathrm{m}$ membrane filtration and subsequent centrifugation. Modified Wadowsky Yee and Glycin,Vacomycin, Polymyxin, Cycloheximide agar plates were both seeded with $0.5 \mathrm{ml}$ water directly from the sample before concentration, $0.1 \mathrm{ml}$ after filtration and $0.1 \mathrm{ml}$ after centrifugation Plates were incubated at $37^{\circ} \mathrm{C}$ for seven to 10 days before colonies were counted. The highest colony count from any of the three steps was considered the result and expressed as colony-forming units (CFU) per litre.

Isolates from water were analysed as isolates from clinical samples, but only two environmental isolates of Legionella pneumophila serogroup 1 subgroup Philadelphila from two different samples were selected for further DNA typing as described above. Isolates (>10) identified as Legionella species by OXOID where identified to species level by sequencing of the Legionella mip gene [15].

\section{Control measures}

To control the Legionella contamination of the hot water system, two interventions were conducted. The first was initiated on 16 January 2009 (11 days after the diagnosis of Case 2) when the temperature and the flow of the water system were increased. On 20 January, water in the boiler was heat-treated at $70^{\circ} \mathrm{C}$ for 12 hours together, after which all residents were requested to flush their taps for five minutes. Subsequently, the water in the boilers was completely replaced with fresh water and the temperature was reduced to $60^{\circ} \mathrm{C}$ for three weeks. Circulation pumps were set at maximum flow.

The second intervention was performed on 10-11 February 2009. For 24 hours the water in the boilers was heated to approximately $70^{\circ} \mathrm{C}$ and all taps were flushed for five minutes. The hot water temperature in the taps was kept at a minimum of $65^{\circ} \mathrm{C}$. The boilers were hyperchlorinated and the temperature was set at $65^{\circ} \mathrm{C}$. All shower hoses in all apartments were replaced with new ones and over the next month the boiler temperature was regulated to ensure the water in the most distant taps was kept at $50^{\circ} \mathrm{C}$. To monitor how the second heat treatment affected the Legionella level in the long term, samples were collected one week, six weeks and seven months after the intervention.

The design, dimensions and regulation of the hot water system, including boilers, pumps, valves and control procedures were evaluated by consulting engineers.

\section{Information to residents}

The residents were informed about the Legionella colonisation of the water system by letters delivered to each apartment on 15, 20 and 21 January 2009. In addition, posters were displayed on the entrance doors of the building, and an information meeting for residents and visitors was organised on 4 February 2009 by the administration . Residents and visitors to the block of flats who had symptoms compatible with Legionella infection (influenza-like symptoms and/or respiratory symptoms) were asked to contact their general practitioner for consultation and collection of samples for laboratory testing.

\section{Results}

Water samples

The sample collected after flushing (B-samples) from the tap in the apartment of Case 2 revealed a hot water temperature below $50{ }^{\circ} \mathrm{C}\left(46{ }^{\circ} \mathrm{C}\right.$ after 15 minutes of flushing) and $5.5 \times 10^{4}$ Legionella CFU/L by culture (Table 1) whereas the temperature should be above $50{ }^{\circ} \mathrm{C}$ as a minimum.

The apartment of Case 2 was situated far from the boilers (only two apartments were placed further away in that direction). The first flush samples (A-samples) collected from shower hose in the apartment of Case 2 showed more than $6 \times 10^{5} \mathrm{CFU} / \mathrm{L}$. This shower hose had rarely been in use, so water had been stagnant for several days. L. pneumophila serogroup 1 subgroup Philadelphia was found in both the A-sample and in the B-sample tapped after 15 minutes. One B-sample was collected from a tap in an apartment very close to the boilers; the temperature measured $56{ }^{\circ} \mathrm{C}$ and only $2 \times 10^{2} \mathrm{CFU} / \mathrm{L}$ were detected in that sample. Only L. pneumophila serogroup 3 was found in the sample. The subgroup Philadelphia isolated from the patient's apartment was also found to be ST 1.

\section{The water system}

During the investigation to reveal the cause of the low hot water temperature in the apartment of Case 2, operational problems were detected. These problems were caused by a combination of low flow in the hot water system and inadequate temperature in the boilers. The circulation pump was adjusted to low capacity, which made the circulation slow. The slow circulation was also caused by small pipe dimensions. In fact the resistance in the pipes was so high that the water was prevented from circulating at the required flow. The slow circulation caused heat loss, and despite the thermostatic adjusting valves being opened, a circulation speed high enough to compensate for the heat loss could not be obtained. Thus, the temperature of the water leaving the boilers was not high enough to compensate for the low flow and the heat reduction throughout the water system. The monitoring arrangements were problematic as well, since a thermometer installed to manually control the water temperature of the hot water return, was found not to function as it showed too high a temperature.

The high concentration of Legionella in some parts of the hot water system resulted in the first intervention which unfortunately failed to eradicate Legionella from the hot water system. The concentration of Legionella decreased in the hot water B-samples (taps), but they remained present, with an average of more than $3 \times 10^{3}$ 


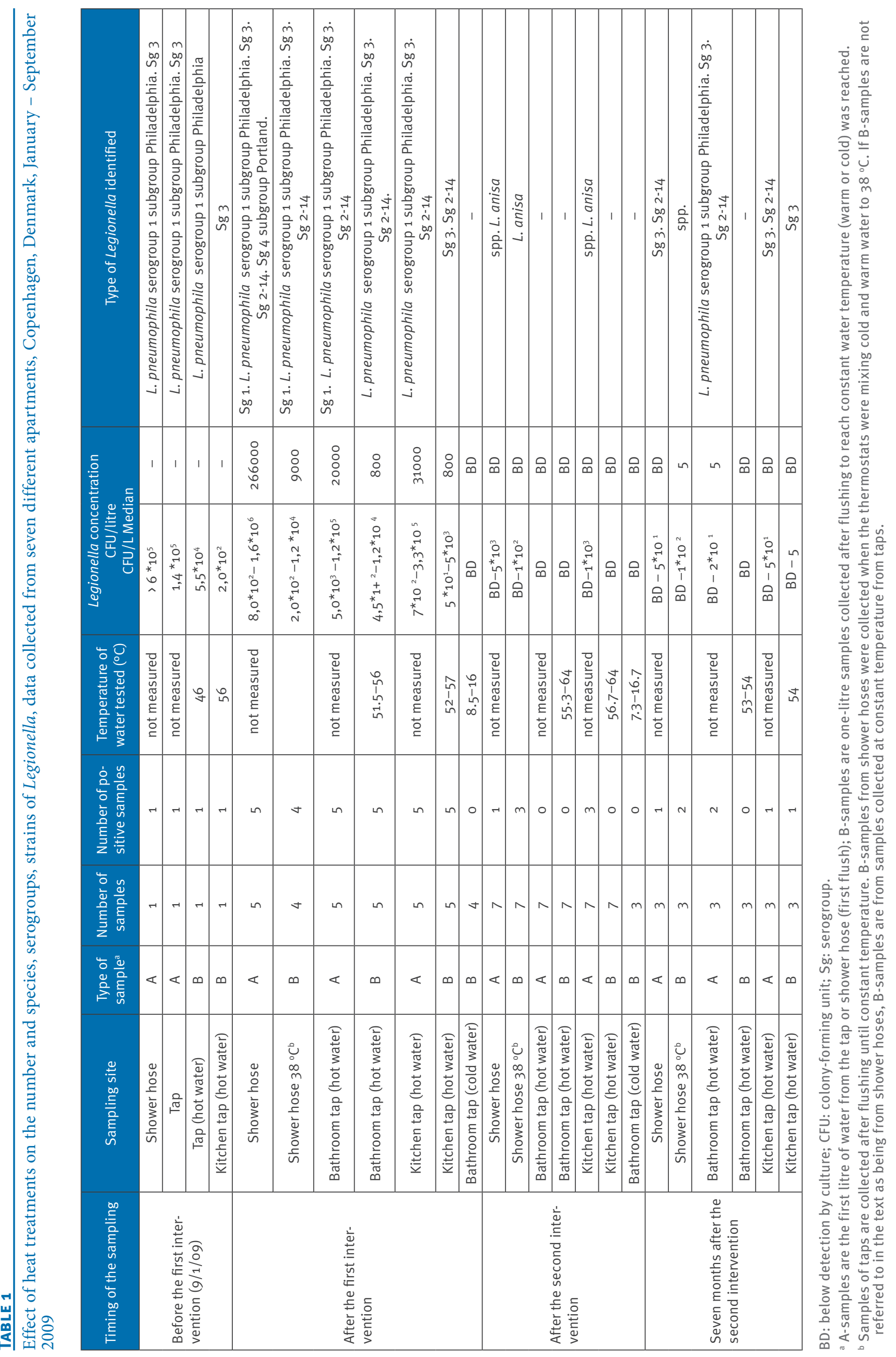




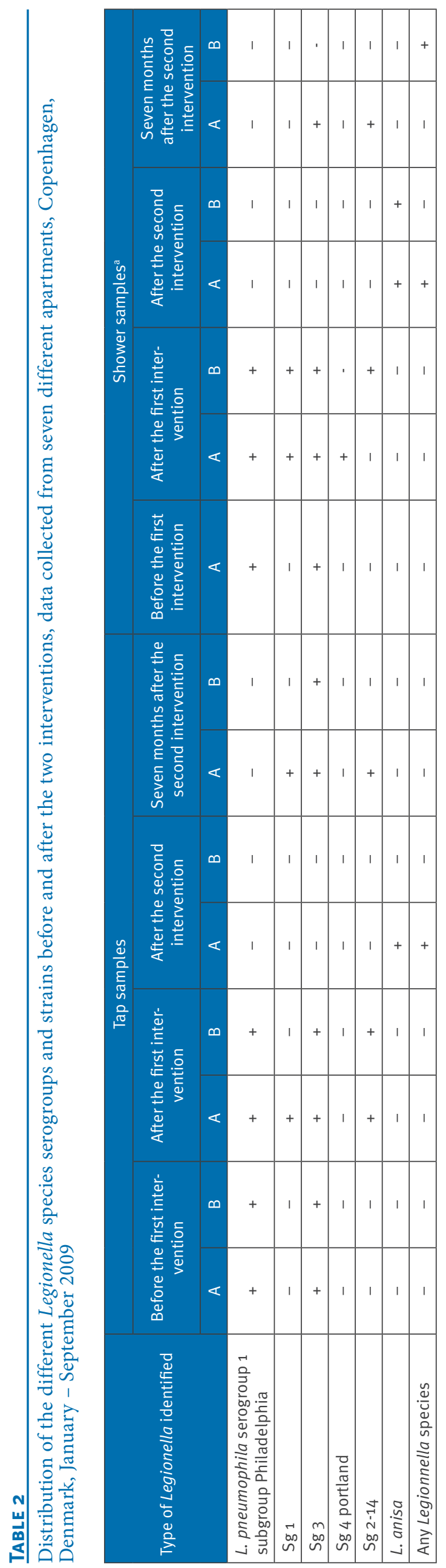

CFU/L (Figure 1). In the A-samples (first flush) from shower hoses, the number of Legionella was high: 8.0 $\times 10^{3}$ to $8.8 \times 10^{6} \mathrm{CFU} / \mathrm{L}$ (Figure 2).

Since Legionella remained present in the water system after the first intervention, a second intervention was conducted. Samples were collected one week (day 38) and six weeks after the second heat treatment (day 73 and 74) and revealed none or only very few Legionella in samples collected from the taps after flushing to constant temperature (B-samples). This indicated that the increased temperature suppressed Legionella growth in the circulating water. However, Legionella remained present in some A-samples although in low numbers. Of seven samples from shower hoses, only one contained Legionella ( $5 \times 10^{3} \mathrm{CFU} / \mathrm{L}$ ) (Table 1). The detection of Legionella in A-samples but not in B-samples, from the same tap or shower hose, indicated local growth. Local growth can be established when the most distant parts of the pipework (shower hose or tap) have not been effectively included in an intervention or if this habitat is particularly favoured for rapid regrowth. Legionella was not detected in the cold water system probably because water temperatures (less than $20^{\circ} \mathrm{C}$ ) were outside the optimal growth temperature for Legionella (Table 1).

Seven months after the second heat treatment, only a few Legionella (five Legionella CFU/L in one sample) were detected by culture of B-samples from taps. Only $50 \mathrm{CFU} / \mathrm{L}$ were detected in one of three A-samples from shower hoses. The second heat treatment and the continuing flow and temperature regulation seemed to control the number of Legionella in this newly built block of flats.

The composition of Legionella species and serogroups changed during the course of the treatments (Table 2).

Before and after the first heat treatment only L. pneumophila was detected (dominated by serogroup 3 and serogroup 1 subgroup Philadelphia) in both Aand B-samples but after the second heat treatment only L. anisa was detected, and mainly in A-samples. Seven months after the second heat treatment, L. pneumophila serogroup 3 and serogroup 1 subgroup Philadelphia were found again, but serogroup 1 Philadelphia was only detected in one A-sample, at a very low ( $5 \mathrm{CFU} / \mathrm{L}$ ) concentration.

\section{Discussion and conclusion}

The hot water in the building was not kept at temperatures outside the range within which Legionella can multiply. Two interventions were conducted to eradicate the Legionella contamination but only the second intervention (water in the boilers heated to $70{ }^{\circ} \mathrm{C}$ for 24 hours followed by $65{ }^{\circ} \mathrm{C}$ for three weeks) followed by a generally increased temperature of the whole warm water system compared to before any of the interventions.was effective with only very limited regrowth after seven months. Other studies [16-18] 
have investigated different kinds of heat treatments but none of them have proven to be effective over a longer period of time. These studies showed that an important factor common to all treatments was that the normal day-to-day operation of the water systems was not adjusted after the different interventions. In this case permanent changes were made in the functioning of the water system after the second heat treatment (higher circulation speed and flushing in unoccupied apartments).

Bacterial biofilms are important for the survival of Legionella and may limit the effectiveness of any intermittent systemic disinfection regime [18]. If not totally erased they constitute a serious factor for potential regrowth. The change we found in the composition of Legionella species before and after the different heat treatments indicated a higher heat tolerance for L. anisa than for L. pneumophila as only L. anisa was cultured immediately after the second heat treatment. Another explanation for this change could be a faster colonisation of $L$. anisa than other Legionella species. When investigating the composition of species it was also shown that L. pneumophila either had survived in the biofilm or had been supplied with the water from the waterworks since this species was detected seven months after the second heat treatment when the temperature was lowered. The finding of L. pneumophila serogroup1 subgroup Philadelphia emphasises the importance of keeping the water system under strict temperature control. L. pneumophila serogroup $1 \mathrm{sub-}$ group Philadelphia was not found in water with temperatures above $55^{\circ} \mathrm{C}$.

L. anisa, which was detected right after the second heat treatment, is common in Danish residential water systems [19], but has only very rarely been associated with infections in humans) [20]. . To get a real picture of the risk of a given water system, it is important to be able to discriminate between different species and serogroups. In this specific cluster, we would probably not have seen any LD cases had L. pneumophila serogroup 1 subgroup Philadelphia not been part of the Legionella flora in the residential area. The subgroup Philadelphia belongs to a virulent subgroup of L. pneumophila serogroup 1 (called Pontiac or MAb 3/1 positive) [21], which is seldom cultured from hot water systems ( $5 \%$ in Denmark) [20].

L. pneumophila serogroup 1 subgroup Philadelphia ST 1 is uncommon in hot water systems. The finding of this particular strain in both patients and in the water system of the new block of flats where both cases had lived or spent time during the incubation period, clearly points to the water as the infectious route.

Legionella is often found in private houses and apartments $[11,23,24]$. In old buildings with old water installations, the risk of Legionella contamination is normally considered to be larger compared with newer buildings with newly established water systems $[10,11,25]$.
However, this cluster demonstrated that newly built blocks of flats can present a risk of Legionella infection. From when a building is finished and water is let into the system until all apartments are inhabited, water may be stagnant in the pipes at ambient temperature, and a biofilm with Legionella can be established in the system. This was probably the situation in the apartment of Case 1. In order to prevent high levels of Legionella in the water pipe systems in new buildings, standard procedures to clean the systems should be applied before occupation. Treatment with biocides could be a solution. In a newly built residential area with many unoccupied apartments, it should also be taken into consideration that the water consumption (both cold and hot water) is lower than the consumption the system is designed for. Hence the water system should be designed to accommodate varying levels of water consumption.

Shower hoses were found to be important risk factors in this study since we found a high number of Legionella in them. This may be due to the material of the hose, temperature and flow of the water. If not regularly flushed with hot water, the low temperature and stagnancy of water in them could pose a risk for infection.

Another risk factor - obvious but nevertheless often overlooked, as in this newly built block of flats is the control and regulation of the water system. Thermostatic heating systems should be properly controlled and correctly sized, including adequate boilers and pumps to run the system optimally. Circulation pumps should have the capacity to keep the water circulating sufficiently also during periods of low water consumption, when the circulation pumps provide the main force in circulating the water. Water should leave the boiler at a temperature hot enough to maintain the temperature above $50{ }^{\circ} \mathrm{C}$ even at the most distant tap and in the return water. Pipe dimensions should be scaled according to the size of the building and flow should be adjustable according to the water consumption.

In the building described in this study, some of the pipes have been changed in order to reduce the resistance. The water system has now two separate re-circulation systems each with a pump, and taps in the apartments that are not occupied are flushed once a week.

\section{Acknowledgements}

We would like to thank Torben Dolin, Department of Environmental Engineering, Technical University of Denmark, for his help during the graphical process. Furthermore we would like to thank KAB for practical assistance during water sampling. LHK was partly financially supported by Graduate School UrbanWaterTech. 


\section{References}

1. World Health Organization (WHO). Legionella and the prevention of leginellosis. Geneva:WHO; 2007. Available from: http://www.who.int/water_sanitation_health/emerging/ legionella.pdf

2. Declerck P, Behets J, van Hoef V, Ollevier F. Detection of Legionella spp. and some of their amoeba hosts in floating biofilms from anthropogenic and natural aquatic environments. Water Res. 2007;41(14):3159-67.

3. Castilla J, Barricarte A, Aldaz J, Garci Cenoz M, Ferrer T, Pelaz C, et al. A large Legionnaires' disease outbreak in Pamplona, Spain: early detection, rapid control and no case fatality. Epidemiol Infect. 2008;136(6):823-32.

4. Ferré MR, Arias C, Oliva JM, Pedrol A, Garcia M, Pellicer T, et al. A community outbreak of Legionnaires' disease associated with a cooling tower in Vic and Gurb, Calalonia (Spain) in 2005. Eur J Clin Microbiol Infect Dis. 2009;28(2):153-9.

5. Benkel DH, McClure EM, Woolard D, Rullan JV, Miller GB Jr, Jenkins SR, et al. Outbreak of Legionnaires' disease associated with a display whirlpool spa. Int J Epidemiol. 2000;29(6):1092-8.

6. Den Boer JW, Yzerman EPF, Schellekens J, Lettinga KD, Boshuizen HC, Van Steenbergen JE, et al. A large outbreak of Legionnaires disease at a flower show, the Netherlands, 1999. Emerg Infect Dis. 2002;8(1):37-43.

7. Ozerol IH. Bayraktar M, Cizmeci Z, Durmaz R, Akbas E, Yildirim Z, et al. Legionnaires' disease: a nosocomial outbreak in Turkey. J Hosp Infect. 2006;62(1):50-7.

8. Unit for Surveillance and Control of Communicable Diseases. Legionnaires' disease in a neonatal unit of a private hospital, Cyprus, December 2008: preliminary outbreak report. Euro Surveill. 2009;14(2):pii=19090. Available from: http://www. eurosurveillance.org/ViewArticle.aspx?Articleld=19090

9. Ricketts K, Joseph CA, Yadav R, on behalf of the European Working Group for Legionella Infections. Travel-associated Legionnaires' disease in Europe in 2008. Euro Surveill. 2010;15(21): pii=19578. Available from: http://www. eurosurveillance.org/ViewArticle.aspx?Articleld =19578

10. Borella P, Montagna MT, Romano-Spica V, Stampi S, Stancanelli G, Triassi M, et al. Leginella infection risk from domestic hot water. Emerg Infect Dis. 2004;10(3):457-64.

11. Mathys W, Stanke J, Harmuth M, Junge-Mathys E. Occurrence of Legionella in hot water systems of single-family residences in suburbs of two German cities with special reference to solar and district heating. Int J Hyg Environ Health. 2008;211(1-2):179-85.

12. Joseph CA, Ricketts KD. on behalf of the European Working Group for Legionella Infections. Legionnares disease in Europe 2007-2008 Euro Surveill. 2010;15(8):pii=19493 Available from: http://www.eurosurveillance.org/ViewArticle.aspx? Articleld $=19493$

13. Helbig JH, Kurtz JB, Pastoris MC, Pelaz C, Lück PC. Antigenic lipopolysaccharide components of Legionella pneumophila recognized by monoclonal antibodies: possibilities and limitations for division of the species into serogroups. J Clin Microbiol. 1997;35(11):2841-5.

14. Ratzow S, Gaia V, Helbig JH, Fry NK, Lück PC. Addition of neuA, the gene encoding $\mathrm{N}$-acylneuraminate cytidylyl transferase, increases the discriminatory ability of the consensus sequence-based scheme for typing Legionella pneumophila serogroup 1 strains. J Clin Microbiol. 2007;45(6):1965-8.

15. Ratcliff RM, Lanser JA, Manning PA., Heuzenroeder MW. Sequence-based classification scheme for the genus Legionella targeting the mip gene. J Clin Microbiol. 1998;36(6):1560-7.

16. Mietzner S, Schwille RC, Farley A, Wald ER, Ge JH, States SJ, et al. Efficacy of thermal treatment and copper-silver ionization for controlling Legionella pneumophila in high -volume hot water pluming systems in hospitals. Am J Infect Control. 1997;25(6):452-7.

17. Steinert M, Ockert G, Lück C, Hacker J. Regrowth of Legionella pneumophilla in heat-disinfected plumbing system. Zentralbl Bakteriol. 1989;288(3):331-42.

18. Mouchtouri V, Velonakis E, Hasjichristodoulou C. Thermal disinfection of hotels, hospitals and athletic venues hot water distribution systems contaminated by Legionella species. Am J Infect Control. 2007;35(9):623-7.

19. Olsen CW, Kjærgaard M, Nielsen SS, Uldum SA. Occurrence of Legionella non-pneumophila in Denmark. Abstract p 93; in Legionella 2009, Institut Pasteur, France, 13-17 October 2009.

20. Bornstein N, Mercatello A Marmet D Surgot M Deveaux $Y$ Fleurette J. Pleural infection caused by Legionella anisa J Clin Microbiol 1989; 27 (9): 2100-2101
21. Helbig JH, Lück PC, Knirel YA, Witzleb W, Zähringer U. Molecular characterization of a virulence-associated epitope on the lipopolysaccharide of Legionella pneumophila serogroup 1.Epidemiol Infect. 1995;115(1):71-8.

22. Pringler N, Brydov $P$, Uldum SA. Occurrence of Legionella in Danish hot water systems. In R Marre, et al. editors. Legionella. 2002;298-301.

23. Arnow PM, Weil D, Para MF. Prevalence and significance of Legionella pneumophila contamination of residential hot-tap water systems. J Infect Dis. 1985;152(1):145-51.

24. Leoni E, De Luca G, Legnani PP, Sacchetti R, Stampi S, Zanetti F. Legionella waterline colonization: detection of legionella species in domestic hotel and hospital hot water systems. J Appl Microbiol. 2005;98(2):373-9.

25. Borella P, Montagna MT, Stampi S, Stancanelli G, RomanoSpica V, Triassi M, et al. Legionella contamination in hot water of Italian Hotels. Appl Environ Microbiol. 2005;71(10):5805-13.

26. International Organization for Standardization. Water quality, Microbiological methods, Detection and enumeration of Legionella. Standard No. ISO 11731:1998. 1998; Geneva, Switzerland. 